\title{
TPH, SLC6A2, SLC6A3, DRD2 and DRD4 Polymorphisms and Neuroendocrine Factors Predict SSRIs Treatment Outcome in the Chinese Population with Major Depression
}

\author{
Authors \\ Affiliations \\ Key words \\ - depression \\ neuroendocrine factors \\ - genes \\ - DRD4 \\ pharmacogenetics
}

\author{
L. Yin ${ }^{1,3}$, Y. Y. Zhang ${ }^{1,3}$, X. Zhang ${ }^{1}$, T. Yu ${ }^{1,2}$, G. He ${ }^{2}$, X. L. Sun
}

Affiliation addresses are listed at the end of the article $\begin{array}{ll}\text { received } & 03.10 .2014 \\ \text { revised } & 07.12 .2014 \\ \text { accepted } & 08.12 .2014\end{array}$

Bibliography

DOI http://dx.doi.org/

10.1055/s-0034-1398508

Published online:

February 2, 2015

Pharmacopsychiatry 2015;

48: 95-103

(c) Georg Thieme Verlag KG

Stuttgart · New York

ISSN 0176-3679

Correspondence

\section{Prof. X. Sun}

Department of Psychiatry

West China Hospital of Sichuan

University

Chengdu

Sichuan Province 610041

People's Republic of China

sunxueli58@163.com

\section{Dr. T. Yu}

Bio-X Institute

Key Laboratory for the

Genetics of Developmental

and Neuropsychiatric Disorders

(Ministry of Education)

Shanghai Jiao Tong University

Shanghai 200030

People's Republic of China

yutao03d@gmail.com

\section{Abstract}

$\nabla$

Objective: This study was intended to determine whether antidepressant outcome to SSRIs was associated with catecholamine gene polymorphisms and neuroendocrine factors in patients of Chinese Han ethnicity with MDD.

Method: A total of 290 MDD patients were recruited and received a 6 -week randomized double-blinded treatment. Cortisol, adrenocorticotropic hormone $(\mathrm{ACTH})$, thyroid-stimulating hormone (TSH), 3'-triiodothyronine (T3), thyroxine (T4), free triiodothyronine (fT3), and free thyroxine (fT4) levels were measured at the baseline. Allele, genotype, and haplotype frequencies of catecholamine genes were compared between responders and non-responders, remission and non-remission groups respectively.

Results: We found that genotype frequency of the rs1800544 polymorphism in the DRD4 gene

\section{Introduction}

\section{$\nabla$}

In a clinical setting, selective serotonin reuptake inhibitors (SSRIs) are the most frequently prescribed antidepressant drugs for major depressive disorder (MDD) treatment. Although these antidepressants have proven to be effective [1], the response and remission rates remain unsatisfactory $[2,3]$. This is partly due to the lack of reliable predictors of treatment outcome [4].

Previous studies have shown that genetic variation may partly explain the inter-individual differences in response to antidepressive drugs [5]. Pathway analysis, which is based on the analysis of variants within genes involved in the same biological pathway, appears to be a particularly promising approach in this regard [6]. Along with rapid developments in the discovery of antidepressants targeting the tyrosine and tryptophan metabolism pathways, pharmacogenetic studies was significantly different between responders and non-responders $(\mathrm{P}<0.05)$. Also the frequency of the rs1800544 CG genotype was significantly higher $(\mathrm{P}<0.05)$ in responders $(51.4 \%)$ than that in non-responders (35.8\%). No significant difference was found between responders and nonresponders, remission and non-remission groups in the SNPs polymorphisms in the TPH, SLC6A2, SLC6A3, or DRD2 genes. The combination of all neuroendocrine factors, clinical characteristics and gene polymorphisms predicted $74.8 \%$ of the variation in SSRI response and 65.5\% in SSRI remission.

Conclusion: Polymorphisms of the DRD4 gene were associated with SSRI response in Chinese Han MDD patients. A combination of clinical characteristics, neuroendocrine factors, and gene polymorphisms might be able to predict the outcome to SSRIs.

on related gene polymorphisms have received increased attention. In particular, several studies have evaluated the correlation between polymorphisms in the tyrosine and tryptophan metabolism pathway genes and MDD [7]. However, the correlations between these polymorphisms and antidepressant response show controversial results which has limited their clinical application [8,9]. In this study, we hypothesize that SSRI response or remission is significantly associated with the tyrosine or tryptophan metabolism pathways gene polymorphisms.

Dysregulation of the hypothalamus-pituitaryadrenal (HPA) axis and the hypothalamus-pituitary-thyroid (HPT) axis plays a pivotal role in the pathogenesis of depression [10-12]. Corticotrophin-releasing hormone (CRH), adrenocorticotrophic hormone (ACTH), and cortisol were found to be increased in older depressed patients, 
and are restored to normal levels after amitriptyline treatment [13]. Enhanced activities of thyrotropin-releasing hormone (TRH) and blunted thyroid-stimulating hormone (TSH) responses to TRH are commonly found in depressive patients [14]. Higher serum TSH levels were found to be associated with response to paroxetine in patients with MDD and could effectively predict the response to paroxetine treatment [15]. However, it is still controversial whether alterations in the HPA or HPT axes can predict the outcome to SSRI treatment.

In the current study, we examined the effect of tyrosine and tryptophan metabolism pathway gene polymorphisms on the antidepressant treatment outcome to SSRIs and explored whether neuroendocrine factors in the HPA and HPT axes could predict the SSRI treatment outcome in MDD patients of Chinese Han ethnicity.

\section{Patients and Methods}

\section{Subjects}

The study was approved by the local research ethics committee. Written informed consent was obtained from each patient. A total of 290 depressed patients, aged 18-55 years, fulfilling the Diagnostic and Statistical Manual of Mental Disorders, Fourth Edition criteria for major depressive disorder, with a minimum 17-item Hamilton Depression Rating Scale (HAMD) score of 18 [16], were recruited for this study from April 2005 to September 2006. All the participants were of unrelated (no blood relationship) Chinese Han origin, and shared similar geographic and sociodemographic characteristics ( $\bullet$ Table 1). The diagnosis of each patient was confirmed in a psychiatric examination performed by an experienced and board-certified psychiatrist. Interrater reliability was evaluated using Kappa coefficients (Kappa value $=0.85$ ). Patients were drug naive, or without any antidepressant treatment for at least 2 weeks (fluoxetine for 4 weeks), and none had received electroconvulsive therapy treatment. Patients with other axis I psychiatric disorders, such as schizophrenia, rapid cycling bipolar disorder, dementia, generalized anxiety disorder, obsessive-compulsive disorder, or substance abuse, and those with axis II disorders (including personality disorders), major medical/neurological disorders, abnormal laboratory baseline values, and pregnancy were excluded.

\section{Treatment}

All patients received a 6 -week period of treatment. Patients were randomly selected for treatment with fluoxetine, paroxetine, citalopram, or sertralin. The total dose per day ranged from 20 to $60 \mathrm{mg}$ of floxetine, paroxetine, or citalopram, and from 50 to $150 \mathrm{mg}$ of sertraline. No other psychotropic medications were permitted during the study except an acceptable dosage of benzodiazepine at bedtime for insomnia.

\section{Data collection}

$5 \mathrm{~mL}$ of peripheral venous blood were collected from each participant for genotyping upon enrollment. Patients' conditions were assessed by trained psychiatrists blinded to genotypes, who were responsible for determining a detailed objective protocol for each patient. The HAMD rating scale was used to assess the severity of symptoms at the baseline and then at 1,2, 4, and 6 weeks following the initiation of treatment, respectively. We used a last-observation-carried-forward (LOCF) analysis, missing data were replaced by the last record value. Response was defined as a more than $50 \%$ reduction in the total HAMD score at the end of the $6^{\text {th }}$ week. Patients with a reduction of HAMD scores $\leq 50 \%$ at the end of the $6^{\text {th }}$ week were assigned to the group of non-responders. Remission was defined as a score of $\leq 7$ in the total HAMD score at the end of the $6^{\text {th }}$ week.

Table 1 Demographic and clinical characteristics of patients.

\begin{tabular}{|c|c|c|c|c|c|c|c|c|c|c|c|c|}
\hline \multirow[t]{2}{*}{ Group } & \multicolumn{2}{|c|}{$\begin{array}{l}\text { Responders } \\
\qquad(n=220)\end{array}$} & \multicolumn{2}{|c|}{$\begin{array}{l}\text { Non-responders } \\
\qquad(n=70)\end{array}$} & \multirow[b]{2}{*}{$t / x^{2}$} & \multirow{2}{*}{ P-value } & \multicolumn{2}{|c|}{$\begin{array}{c}\text { Remission } \\
(n=144)\end{array}$} & \multicolumn{2}{|c|}{$\begin{array}{l}\text { Non-remission } \\
\quad(n=146)\end{array}$} & \multirow[b]{2}{*}{$t / x^{2}$} & \multirow{2}{*}{ P-value } \\
\hline & Mean & SD & Mean & SD & & & Mean & SD & Mean & SD & & \\
\hline age (years) & 35.51 & 13.62 & 37.74 & 12.19 & 1.119 & 0.197 & 33.81 & 13.42 & 38.16 & 12.75 & -2.835 & $0.005^{*}$ \\
\hline number of episodes & 1.51 & 0.859 & 1.56 & 1.326 & 5.124 & 0.787 & 1.43 & 0.687 & 1.61 & 1.211 & -1.576 & 0.116 \\
\hline body mass index & 21.90 & 4.37 & 21.98 & 3.57 & 0.023 & 0.891 & 21.44 & 3.49 & 22.37 & 4.77 & -1.849 & 0.066 \\
\hline HAMD baseline & 24.42 & 4.89 & 24.37 & 4.82 & -0.070 & 0.944 & 23.43 & 4.44 & 25.37 & 5.09 & -3.455 & $0.001^{*}$ \\
\hline HAMD-6week & 5.96 & 3.46 & 18.33 & 7.69 & 18.640 & $0.000^{*}$ & 3.90 & 2.09 & 13.38 & 6.47 & -16.719 & $0.000^{*}$ \\
\hline gender & $\mathrm{N}$ & $\%$ & $\mathrm{~N}$ & $\%$ & 0.229 & 0.992 & $\mathrm{~N}$ & $\%$ & $\mathrm{~N}$ & $\%$ & 0.499 & 0.481 \\
\hline male & 107 & 48.6 & 34 & 48.6 & & & 67 & 46.5 & 74 & 50.7 & & \\
\hline female & 113 & 51.4 & 36 & 51.4 & & & 77 & 53.5 & 72 & 49.3 & & \\
\hline marital status & $\mathrm{N}$ & $\%$ & $\mathrm{~N}$ & $\%$ & 0.622 & 0.522 & $\mathrm{~N}$ & $\%$ & $\mathrm{~N}$ & $\%$ & 6.756 & $0.011^{*}$ \\
\hline single (never married) & 82 & 37.3 & 21 & 30.0 & & & 63 & 43.75 & 40 & 27.4 & & \\
\hline married & 122 & 55.5 & 44 & 62.9 & & & 72 & 50.0 & 94 & 64.38 & & \\
\hline divorced or remarried & 16 & 7.2 & 5 & 7.1 & & & 9 & 6.25 & 12 & 8.22 & & \\
\hline family history & $\mathrm{N}$ & $\%$ & $\mathrm{~N}$ & $\%$ & 2.919 & 0.094 & $\mathrm{~N}$ & $\%$ & $\mathrm{~N}$ & $\%$ & 0.421 & 0.517 \\
\hline yes & 29 & 13.2 & 15 & 21.4 & & & 23 & 15.6 & 21 & 14.7 & & \\
\hline no & 191 & 86.8 & 55 & 78.6 & & & 124 & 84.4 & 122 & 85.3 & & \\
\hline \multicolumn{13}{|l|}{ neuroendocrine factor } \\
\hline CORT (nmol/L) & 415.76 & 88.92 & 416.68 & 75.18 & 1.015 & 0.937 & 434.73 & 135.88 & 387.72 & 119.93 & 1.764 & 0.081 \\
\hline ACTH (ng/L) & 29.52 & 7.44 & 28.64 & 6.72 & 9.293 & 0.369 & 31.69 & 13.17 & 28.10 & 11.71 & 1.186 & 0.240 \\
\hline TSH (mU/L) & 2.69 & 1.19 & 2.85 & 1.22 & 0.034 & 0.316 & 2.70 & 1.74 & 2.91 & 1.99 & -0.561 & 0.576 \\
\hline fT3 (pmol/L) & 4.49 & 0.52 & 4.42 & 0.39 & 4.535 & 0.340 & 4.41 & 0.75 & 4.45 & 0.90 & -0.246 & 0.806 \\
\hline fT4 (pmol/L) & 15.95 & 2.36 & 15.49 & 1.43 & 8.821 & 0.131 & 16.13 & 3.82 & 15.28 & 3.17 & 1.23 & 0.222 \\
\hline T3 (nmol/L) & 1.62 & 0.16 & 1.62 & 0.04 & 0.713 & 0.909 & 1.56 & 0.31 & 1.64 & 0.34 & -0.951 & 0.346 \\
\hline T4 (nmol/L) & 91.14 & 9.88 & 91.53 & 6.73 & 1.061 & 0.836 & 86.93 & 15.42 & 93.65 & 25.24 & -1.173 & 0.246 \\
\hline
\end{tabular}

HAMD = Hamilton depression rating scale; ${ }^{*} \mathrm{p}<0.05$ 
7 neuroendocrine indicators were evaluated, as follows: cortisol, ACTH, TSH, 3'-triiodothyronine (T3), thyroxine (T4), free triiodothyronine (fT3), and free thyroxine (fT4). Cortisol, T3, T4, fT3, and fT4 were measured by electrochemiluminescene quantitative assays. TSH was measured using an electrochemiluminescene double-antibody sandwich method. ACTH was measured by radioimmunoassay. All of the above indicators were detected with a Roche E170 analytical system in the clinical laboratory of the West China Hospital.

\section{Single nucleotide polymorphism (SNP) selection and genotyping}

Among the many catecholamine genes, TH, DRD2, DRD4, SLC6A2, and SLC6A3 are the most well-studied in MDD. We used the following criteria for SNP selection of these genes: (1) the SNP is in a functional region of the gene; (2) the SNP was shown to have strong effects on drug response in previous studies, especially genome-wide association studies; and (3) it was a tag SNP.

Genomic DNA was extracted from venous blood leukocytes using the standard phenol-chloroform method. Considering that the coverage of a gene and a minor allele frequency occur at a rate above 0.03 , we selected 19 single SNPs for the TH, DRD2, DRD4, SLC6A2, and SLC6A3 genes from the literature and the National Center for Biotechnology Information dbSNP database (http://www.ncbi.nlm.nih.gov/SNP). Of the 19 SNPs, 2 (rs2234689, rs3863145) were downstream, 4 (rs1362621, rs2550948, rs2550956, rs2937639) were upstream, 2 (rs140504, rs6356) were in a coding exon, 4 (rs10743152, rs10770141, rs1800544, rs933399) were in the promoter region, 4 (rs2242446, rs4963126, rs5564, rs7131056) were in introns, 2 (rs2292023, rs27072) were in the 3' untranslated region, and 1 (rs5569) was a synonymous codon. All SNPs were genotyped with a matrix-assisted laser desorption/ionization time-of-flight mass spectrometer using a MassARRAY(R) Analyzer 4 platform (Sequenom; San Diego, CA, USA). All primers were designed by the accompanying software, Spectrodesigner. Polymerase chain reactions (PCRs) were carried out in a total volume of $5 \mu \mathrm{L}$ with10 ng genomic DNA, under the cycling conditions recommended by the manufacturer. Detailed information about the primers and PCR conditions is available on request. The determination of genotypes was performed by researchers who were blinded to the clinical outcome of the antidepressant treatment.

\section{Statistical analysis}

Demographic and clinical variables were compared using the Student's t test (age, body mass index, HAMD scores, number of episodes, neuroendocrine factors) or Pearson's $\mathrm{X}^{2}$ test (gender, marital status, family history), and Fisher's exact test was used to compare data between responders and non-responders, remission and non-remission groups. A stepwise logistic regression was used to examine the relationship between genotype, neuroendocrine factors, clinical variables and treatment outcome. All tests were 2-tailed and statistical significance was accepted at $\mathrm{P}<0.05$. Statistical analyses were performed using SPSS 17.0. Analysis of allelic and genotypic distributions and pairwise linkage disequilibria were performed using SHEsis software (http://202.120.31.177/myanalysis.php) [17]. The discrepancies in allele and genotype frequency between groups were compared by using a $\mathrm{X}^{2}$ test. Odd ratios (ORs) and their $95 \%$ confidence intervals (CIs) were also calculated. Deviations from Hardy-Weinberg equilibrium were tested using HaploView version 4.2 [18]. Haplotype construction was initially performed on HaploView and further analysis was carried out with SHEsis software [19].

\section{Results}

$\nabla$

A total of 303 patients were initially enrolled in this study. Ten patients were removed due to the absence of blood samples or because genotyping failed due to the poor quality of their blood samples. Furthermore, 3 patients dropped out as a result of intolerable adverse effects at their first or second visit. A cohort of 290 patients was used in the final analysis.

\section{Demographic and clinical characteristics}

Clinical and demographic characteristics of subjects and the levels of significance of differences in clinical variables between responders and non-responders, remission and non-remission groups are shown in 0 Table 1 . Of the 290 patients who completed the 6-week SSRI treatment trial, 220 were responders and 70 were non-responders, 144 were in the remission and 146 were in the non-remission groups, for an overall rate of response of $75.9 \%$ and remission of $49.7 \%$. There were no significant differences in age, gender, body mass index, HAMD scores at baseline, number of previous episodes of MDD, marital status, or family history $(P=0.197,0.992,0.891,0.944,0.787,0.552,0.094$, respectively) between responders and non-responders. There were no significant differences in gender, body mass index, number of previous episodes of MDD, or family history between the remission and non-remission groups $(P=0.481,0.066,0.116$, 0.517). But there were significant differences in age, HAMD scores at baseline, marital status between remission and nonremission groups $(P=0.005,0.001,0.011)$. No significant differences in neuroendocrine levels were found between responders and non-responders, remission and non-remission groups, i.e., CORT, ACTH, TSH, fT4, fT3, T3, and T4 levels ( $P=0.937,0.369$, $0.316,0.131,0.340,0.909$, and 0.836 between responders and non-responders, $\mathrm{P}=0.081,0.240,0.576,0.222,0.806,0.346$ and 0.246 between remission and non-remission groups, respectively).

\section{Genotype and allele frequencies of DRD4}

The genotype and allele frequencies of 14 SNPs in each group are shown in Table 2, 3. 5 SNPs (rs2550948, rs27072, rs2937639, rs5569, and rs933399) were omitted from the analysis because they deviated from the values expected under Hardy-Weinberg equilibrium $(\mathrm{P}<0.001$ for all). The frequency of the DRD4 rs1800544 CG genotype was significantly higher $(\mathrm{P}<0.05)$ in the SSRIs responders $(51.4 \%)$ than that in the non-responders (35.8\%). The frequencies of the DRD4 rs1800544 CC and GG genotypes were significantly lower $(\mathrm{P}<0.05)$ in the SSRIs responders $(7.7 \%, 40.9 \%)$ than that in the non-responders (19.4\%, $44.8 \%)$. A response rate to SSRIs was strongly associated with the genotype of the DRD4 rs1800544 polymorphism (OR=6.38; 95\% $\mathrm{CI}=1.53-18.71 ; \mathrm{P}=0.003$, false discovery rate [FDR] $\mathrm{P}=0.042$ ). Allele frequencies of rs 1800544 polymorphisms and those of other SNPs (TPH, SLC6A2, SLC6A3, and DRD2) did not differ significantly between responders and non-responders $(\mathrm{P}>0.05)$. Referring to the remission and non-remission groups, genotype and allele frequencies did not differ significantly between groups after FDR adjusted $(\mathrm{P}>0.05)$. 


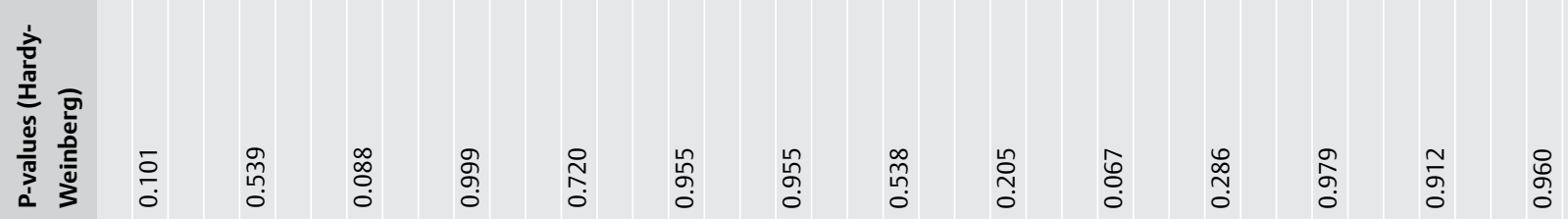

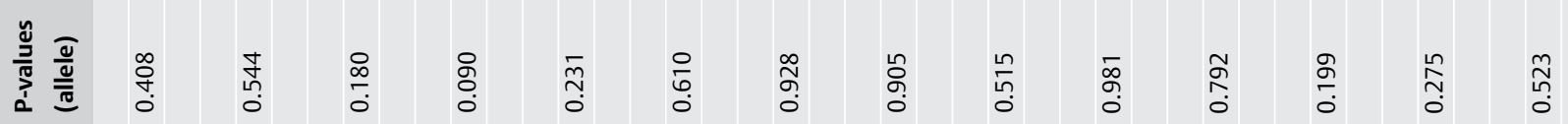

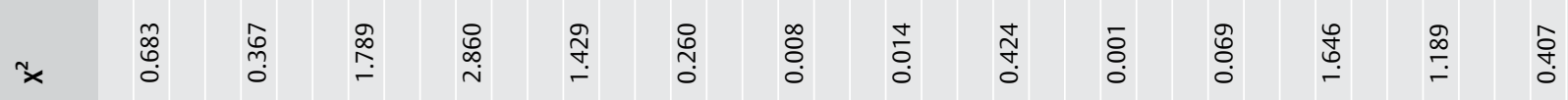

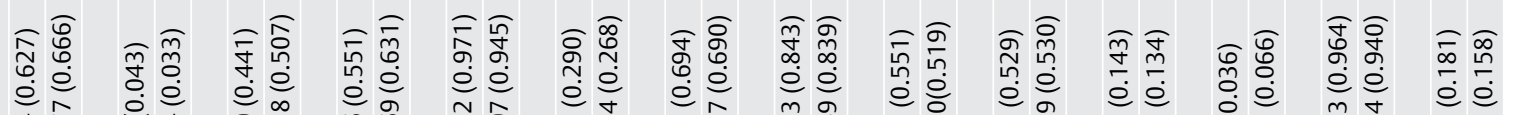

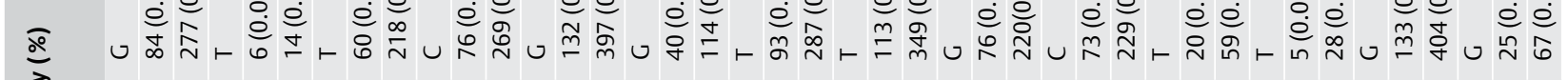

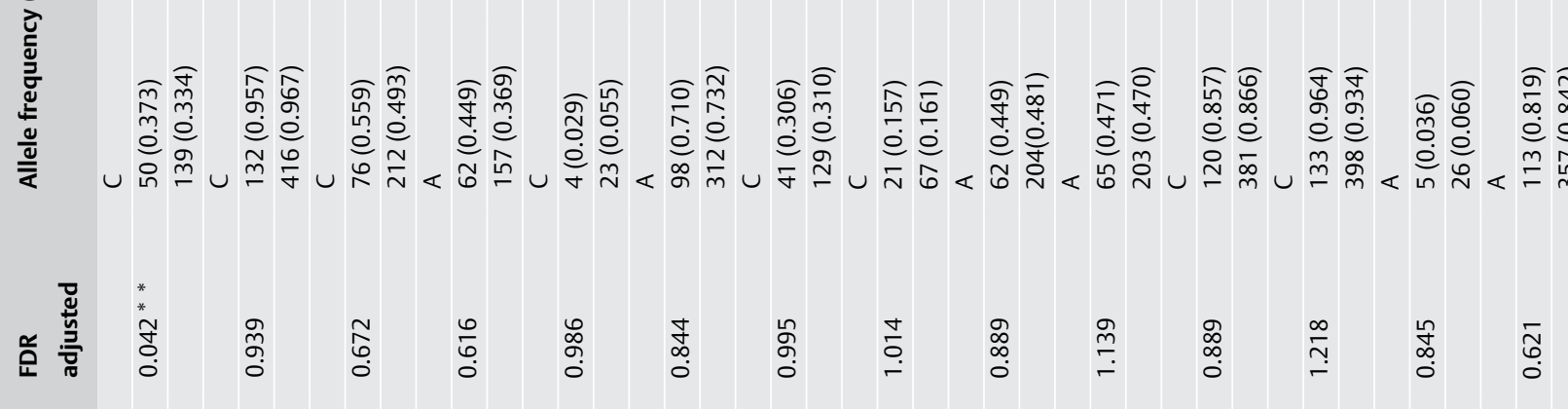

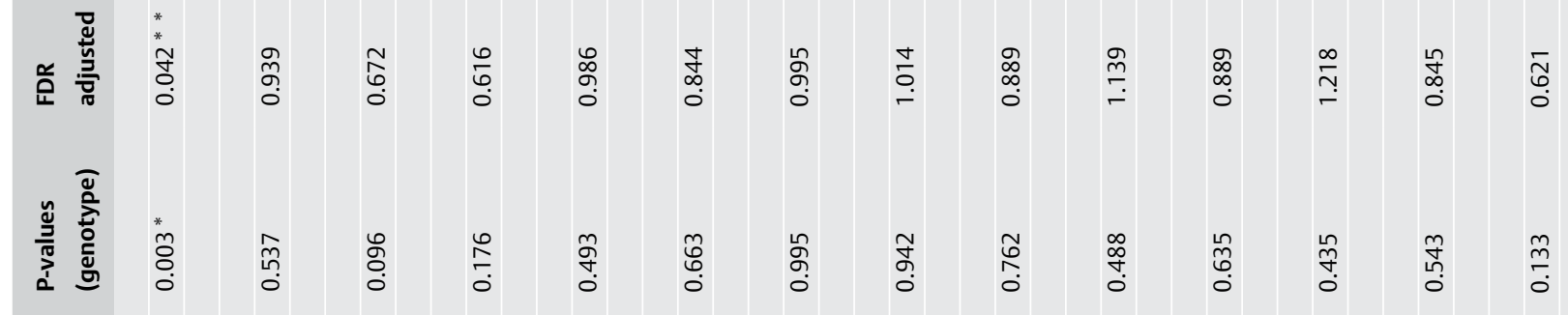

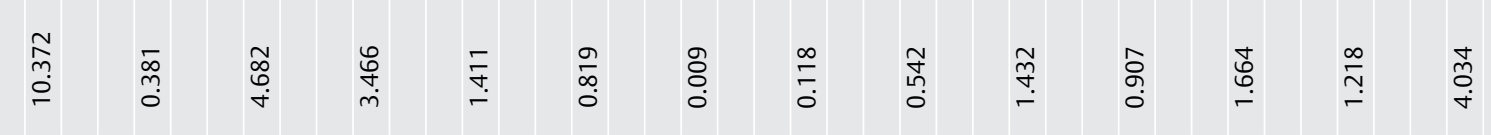

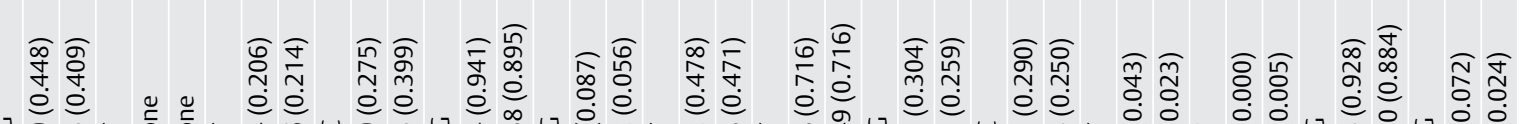

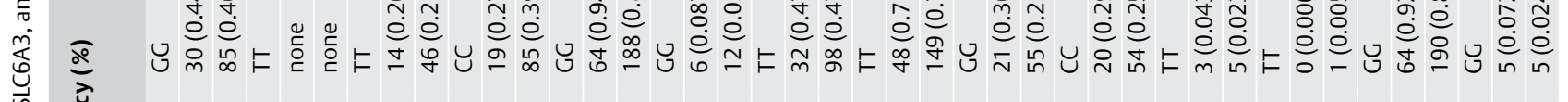

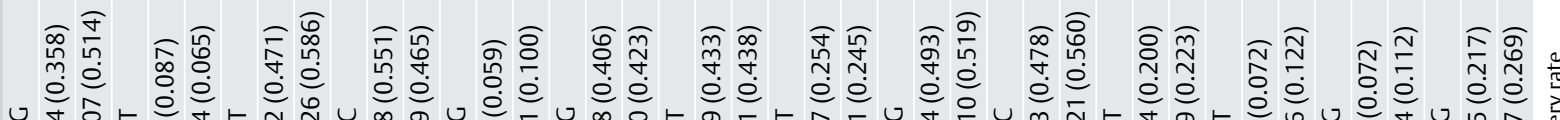
Ud オ

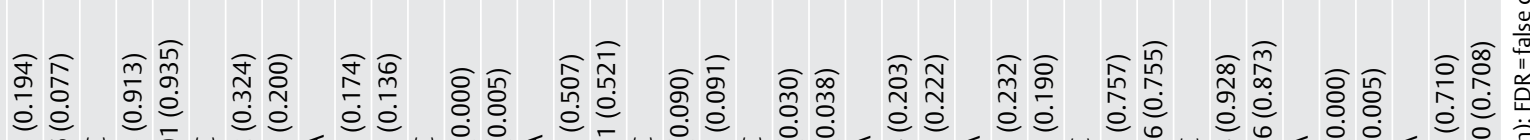

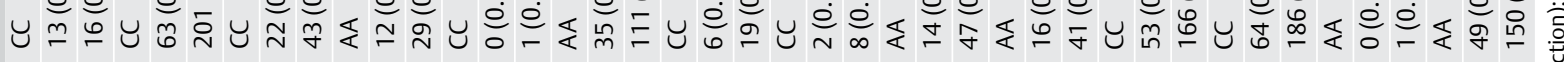

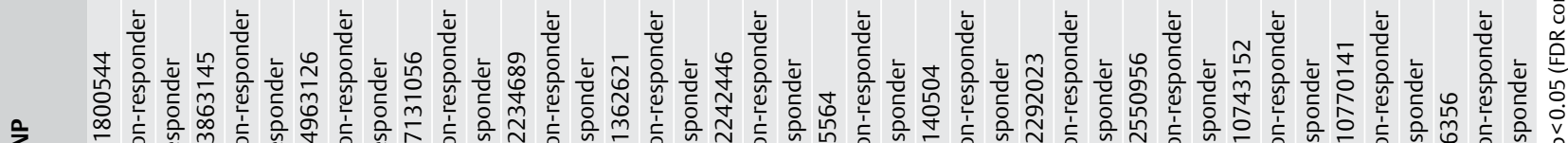

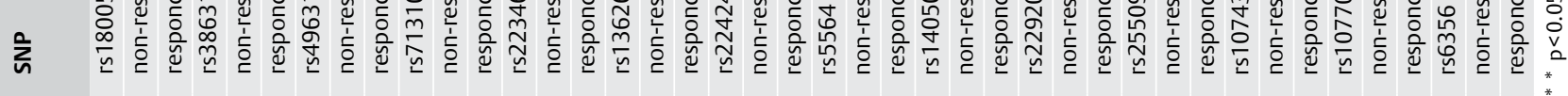

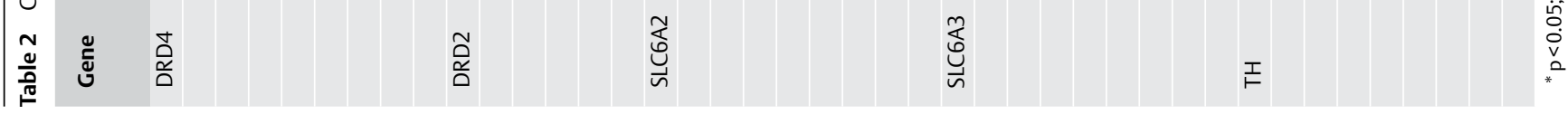


If

高 絗 总

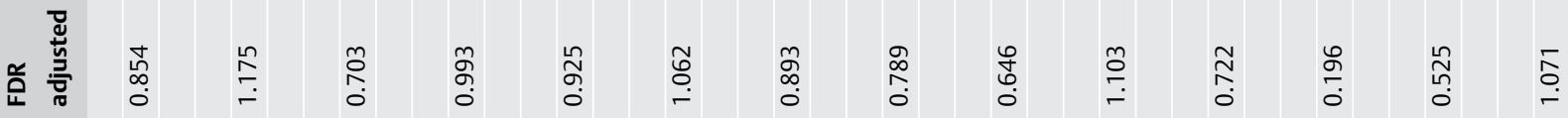

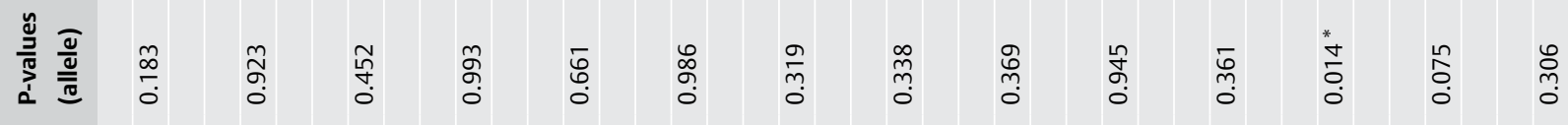

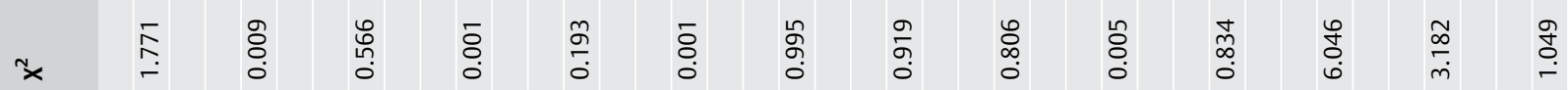

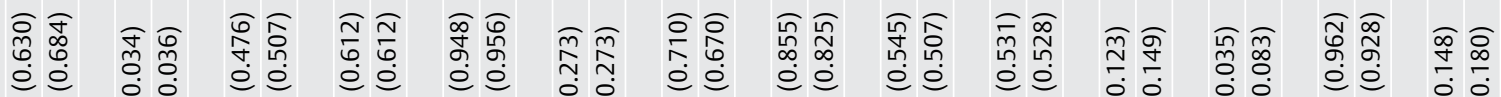
৩

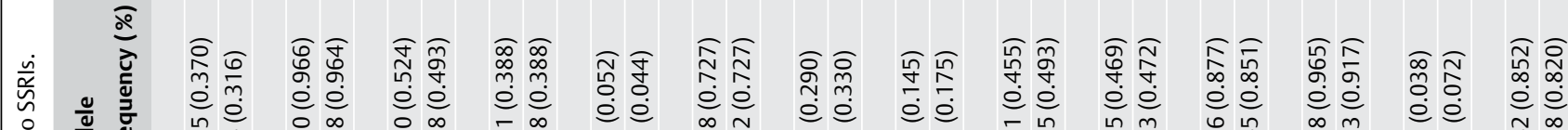

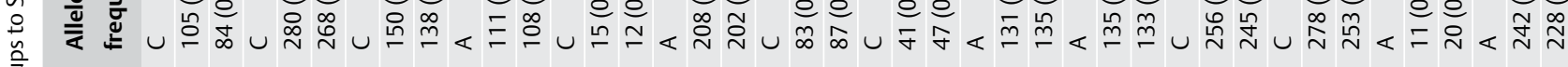

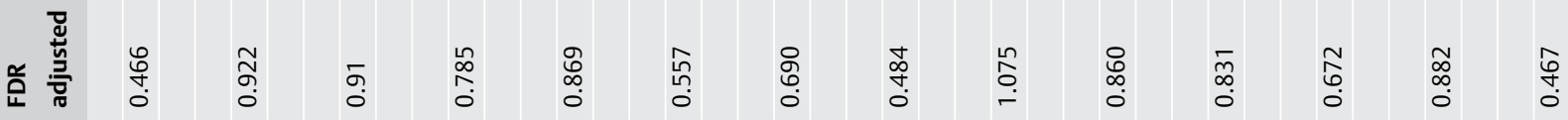

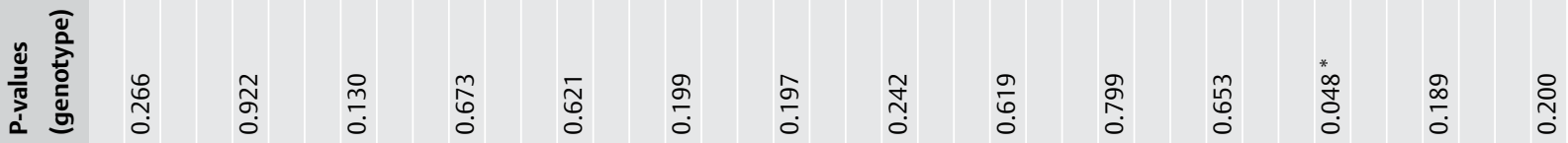

丞

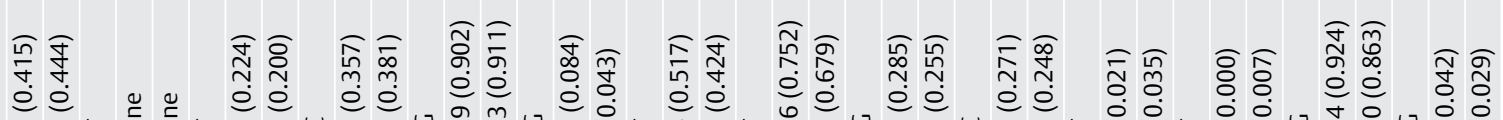

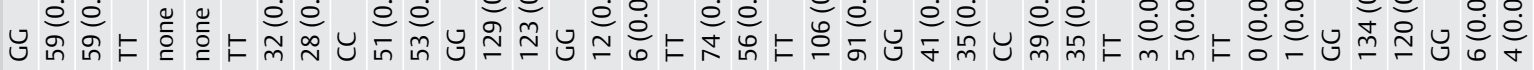

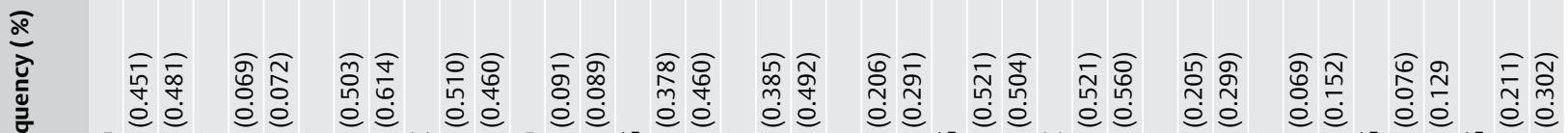

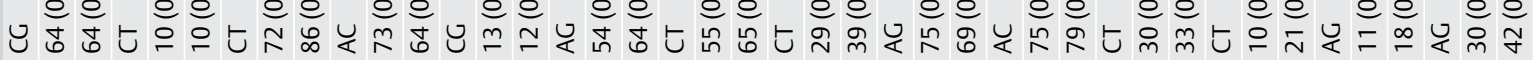

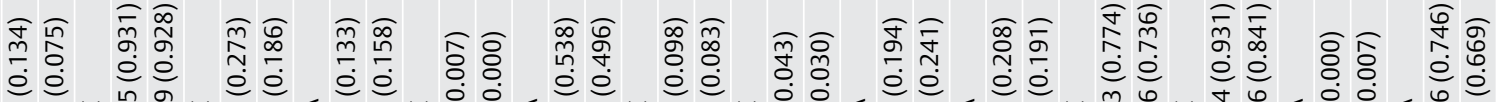

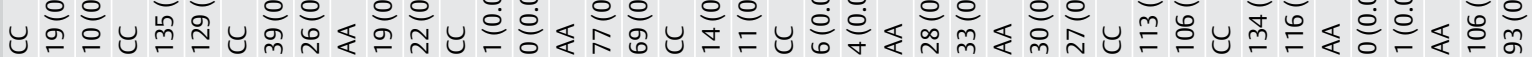

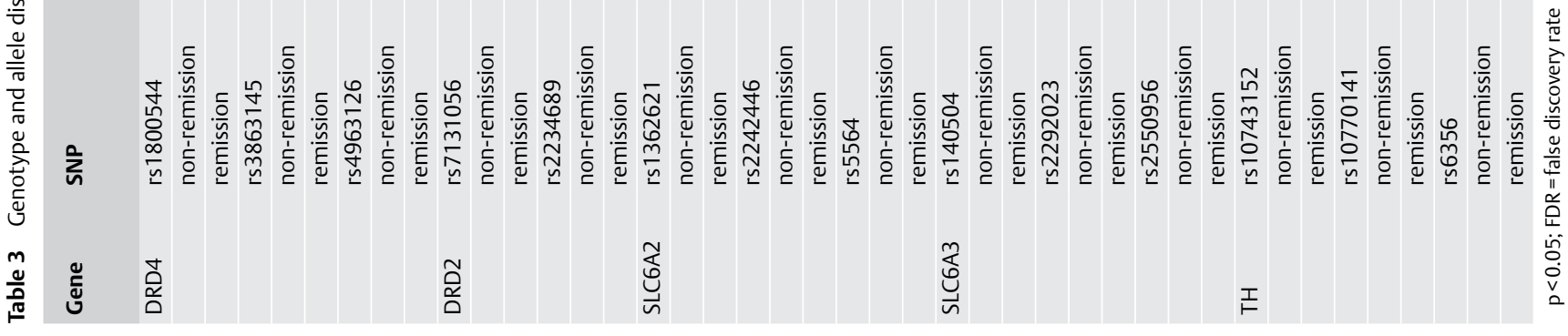




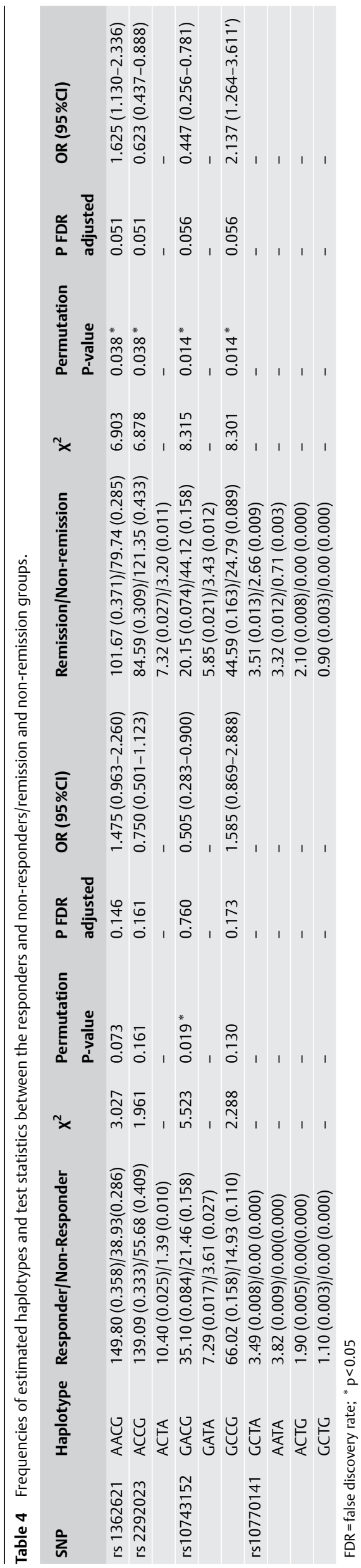

Haplotype frequencies of SNPs between the responders and non-responders

Pairwise linkage disequilibrium (LD) between the 14 markers was conducted. Strong LD was observed between the 2 haplotype blocks composed of rs2292023-rs1362621 ( $D^{\prime}=0.990$; $\mathrm{r}^{2}=0.827 ; \quad$ SLC6A2 gene) and rs10743152-rs10770141 $\left(D^{\prime}=1.000 ; r^{2}=0.904 ;\right.$ TH gene). Therefore, we estimated the haplotype distributions with these SNPs ( $\odot$ Table 4). Haplotypes were removed from this analysis if their estimated frequencies were less than $3 \%$ in either the responder or non-responder group. Neither of these markers remained significant after FDR correction ( $\odot$ Table 4; FDR P>0.05). Thus, no association was found between either of the haplotype blocks and SSRI treatment response at week 6 .

\section{Haplotype frequencies of SNPs between the remission} and non-remission groups

LD between the 14 markers was conducted between the remission and non-remission groups. Strong LD was observed between the 2 haplotype blocks composed of rs2292023rs1362621 $\left(D^{\prime}=0.990 ; r^{2}=0.827\right.$; SLC6A2 gene $)$ and rs10743152rs10770141 $\left(D^{\prime}=1.000 ; r^{2}=0.904 ;\right.$ TH gene $)$. Neither of these markers remained significant after FDR correction ( $\Theta$ Table 4; FDR P>0.05). Thus, no association was found between either of the haplotype blocks and SSRI treatment remission at week 6 .

\section{Clinical characteristics, neuroendocrine factors, gene polymorphisms and treatment outcome}

We used SSRI response or remission as the dependent factor; genotype of rs1800544, age, gender, baseline scores on the HAMD, number of episodes, family history, marital status, and neuroendocrine indicators (CORT, ACTH, TSH, fT3, fT4, T3, and T4) as covariates in the logistic regression ( $\bullet$ Table 5, 6). None of the factors was a significant predictor of SSRI response when examined independently $(P>0.05$, respectively). Baseline scores on the HAMD was a significant predictor of SSRI remission when examined independently $(P=0.015)$. We also found the combination of all factors (genotype of rs1800544, age, gender, baseline scores on the HAMD, number of episodes, family history, marital status, and neuroendocrine factors) predicted $74.8 \%$ of the variation in SSRI response and $65.5 \%$ in SSRI remission (0 Table 6).

\section{Discussion}

In our study we found that the rs 1800544 polymorphisms of the DRD4 gene were significantly associated with MDD response after 6 weeks of SSRI treatment. No neuroendocrine indicator independently predicted the SSRI response or remission in our study; however, a combination of neuroendocrine factors, some clinical characteristics and rs 1800544 polymorphisms predicted $74.8 \%$ of the SSRI response and $65.5 \%$ of the SSRI remission. There were significant differences in age, HAMD scores at baseline, marital status between remission and non-remission groups.

\section{DRD4 gene polymorphisms are associated with SSRIs response}

Our data revealed that the rs1800544 (DRD4 gene) polymorphisms play a major role in the antidepressant response to SSRI treatment. The frequency of the DRD4 rs1800544 CG genotype 
Table 5 The logistic regression in which SSRI response (or remission) was the dependent factor.

\begin{tabular}{|llllllll} 
Covariate & B & S.E. & Wald & df & Sig & Exp (B) & 95\%CI for Exp (B) \\
\hline genotype & $0.188(0.320)$ & $0.311(0.275)$ & $0.363(1.354)$ & 1 & $0.547(0.245)$ & $1.206(0.726)$ & $0.655-2.22(0.424-1.245)$ \\
\hline age & $-0.014(0.002)$ & $0.017(0.015)$ & $0.607(0.025)$ & 1 & $0.436(0.874)$ & $0.986(1.002)$ & $0.953-1.021(0.973-1.032)$ \\
\hline gender & $-0.145(0.360)$ & $0.322(0.280)$ & $0.202(1.659)$ & 1 & $0.653(0.198)$ & $0.865(1.434)$ & $0.460-1.627(0.829-2.480)$ \\
\hline HAMD-baseline & $0.042(-0.074)$ & $0.035(0.030)$ & $1.454(5.951)$ & 1 & $0.228\left(0.015^{*}\right)$ & $1.043(0.929)$ & $0.974-1.117(0.876-0.986)$ \\
\hline number of episodes & $0.052(-0.182)$ & $0.161(0.166)$ & $0.103(1.198)$ & 1 & $0.748(0.274)$ & $1.053(0.834)$ & $0.768-1.444(0.602-1.155)$ \\
family history & $-0.451(-0.225)$ & $0.424(0.386)$ & $1.134(0.340)$ & 1 & $0.287(0.560)$ & $0.637(0.798)$ & $0.278-1.461(0.374-1.702)$ \\
\hline single & $0.598(0.932)$ & $0.654(0.614)$ & $0.836(2.305)$ & 1 & $0.360(0.129)$ & $1.818(2.539)$ & $0.505-6.549(0.762-8.458)$ \\
\hline married & $0.576(0.129)$ & $0.581(0.558)$ & $0.983(0.054)$ & 1 & $0.321(0.817)$ & $1.779(1.138)$ & $0.570-5.552(0.381-3.399)$ \\
\hline divorced & & & $1.090(4.137)$ & 2 & $0.580(0.126)$ & & \\
CORT & $0.003(0.003)$ & $0.003(0.002)$ & $1.234(1.779)$ & 1 & $0.267(0.182)$ & $1.003(1.003)$ & $0.998-1.008(0.998-1.008)$ \\
\hline ACTH & $-0.002(0.038)$ & $0.029(0.027)$ & $0.005(2.092)$ & 1 & $0.946(0.148)$ & $0.998(1.039)$ & $0.943-1.056(0.986-1.094)$ \\
\hline TSH & $-0.214(-0.167)$ & $0.154(0.153)$ & $1.931(1.196)$ & 1 & $0.165(0.274)$ & $0.807(0.846)$ & $0.597-1.092(0.627-1.142)$ \\
\hline fT3 & $-0.171(-0.239)$ & $0.368(0.355)$ & $0.215(0.453)$ & 1 & $0.643(0.501)$ & $0.843(0.787)$ & $0.410-1.734(0.392-1.580)$ \\
\hline fT4 & $0.271(0.238)$ & $0.160(0.191)$ & $2.878(1.546)$ & 1 & $0.090(0.214)$ & $1.311(1.269)$ & $0.959-1.792(0.872-1.846)$ \\
\hline T3 & $0.572(-1.193)$ & $1.283(1.342)$ & $0.199(0.791)$ & 1 & $0.656(0.374)$ & $1.771(0.303)$ & $0.143-21.881(0.022-4.207)$ \\
\hline T4 & $-0.032(-0.039)$ & $0.030(0.032)$ & $1.185(1.526)$ & 1 & $0.276(0.217)$ & $0.968(0.961)$ & $0.913-1.026(0.903-1.023)$ \\
\hline constant & $-1.530(2.060)$ & $2.794(2.430)$ & $0.300(0.719)$ & 1 & $0.584(0.397)$ & $0.217(7.846)$ & \\
\hline
\end{tabular}

Genotype of rs 1800544 was included in logistic regression which included C allele carrier (CG or CC) or not (GG). The values in the brackets are the results of logistic regression in which SSRI remission was the dependent factor

Table 6 The predictive probability of clinical characteristics, neuroendocrine factors and gene polymorphisms in SSRI treatment outcome.

\begin{tabular}{|l|ccc|}
\hline & \multicolumn{3}{l}{ Predicted by combination of all factors } \\
Observed & Negative & Positive & Percentage Correct (\%) \\
\hline non-responder & 11 & 59 & 15.7 \\
\hline responder & 14 & 206 & 93.6 \\
\hline $\begin{array}{l}\text { overall } \\
\text { non-remission }\end{array}$ & 95 & 51 & 74.8 \\
\hline remission & 49 & 95 & 65.1 \\
\hline overall & & & 65.0 \\
\hline
\end{tabular}

All factors include age, gender, HAMD baseline, number of episodes of MDD, family history, marital status, rs 1800544 genotype and neuroendocrine factors. Neuendocrine factors include CORT, ACTH, TSH, fT3, fT4, TT3, TT4. Negative indicate non-response or non-remission to SSRI treatment predicted by logistic regression. Positive indicate response or remission to SSRI treatment predicted by logistic regression. Number of patients or correct percentage are shown

was significantly higher in the SSRI responders (51.4\%) than that in the non-responders (35.8\%). Dopamine (DA) is hypothesized to play an important role in MDD pathogenesis and antidepressant effects. DA function is increased after chronic antidepressant treatment $[20,21]$. Also the antidepressant effects of SSRIs are reversed by acute administration of a $\mathrm{D} 2$ receptor-selective antagonist [22]. Nevertheless, the DA system has been poorly investigated in previous pharmacogenetic studies, which have instead focused on dopamine receptors belonging to the D2-like family (D2, D3, and D4). Garriock et al. reported that a variable number tandem repeat (VNTR) polymorphism within exon 3 of DRD4 may modulate the antidepressant efficacy [23], but no association with SSRI response was found in a larger sample [24]. Our results support our primary hypothesis that SSRI response is significantly associated with the DRD4 gene polymorphisms. Genetic make-up of the Asian people is likely to differ from other ethnic groups, which maybe can partly explain the different outcomes of antidepressant treatment with SSRI relative to previous studies. As this genetic subgroup (CG) comprised $59.5 \%$ of the present cohort (131/220 cases), this result may prove important for clinical practice. This preliminary finding should be further tested in studies specifically designed to examine the differential response to drug class according to genotype.

\section{TPH, SLC6A2, SLC6A3, and DRD2 gene polymorphisms} are not associated with SSRIs response or remission Our data revealed no association between polymorphisms in the TPH, SLC6A2, SLC6A3, and DRD2 genes and the efficacy of SSRIs. The noradrenaline transporter or the norepinephrine transporter, encoded by the SLC6A2 gene, is a primary target of several antidepressant agents. Previous studies reported that the $G$ allele of the SLC6A2 variant rs5569 (G1287A) might be associated with antidepressant outcome, especially for noradrenergic antidepressants, and that the T allele of rs2242446 (T182C) may predict a better milnacipran response $[8,9,25]$. On the other hand, negative findings for the association of both of these polymorphisms and patient response have also been reported [26-29].

The SLC6A3 gene encodes the dopamine transporter DAT, which is a membrane-spanning protein whose primary function is to clear DA from the synapses. A 40-bp VNTR in exon 15 has been reported to affect DAT expression [30]. In addition, the 9/10 and 9/9 SLC6A3 genotypes may be associated with the risk of poorer and slower response to various antidepressants [31] and SSRI augmentation with methylphenidate [32]. Another variant in SLC6A3 (rs8179029) was associated with desipramine response in Mexican-Americans, but confirmation of this result is lacking [25]. Thus, given the low number of available studies, no definitive conclusion of the association between this gene and antidepressant outcome can be established.

Associations between polymorphisms of the TPH gene, which encodes tryptophan hydroxylase, and SSRI response were confirmed by pharmacogenetic studies [33-35], but these results have not been replicated for the most part, especially in Asian populations but also in Caucasians [36-42].

Similarly, studies examining the role of the DRD2 gene in antidepressant response are conflicting. Negative results were obtained for the rs1801028 and rs6275 polymorphisms, while a significant association between early improvement and rs4460839/ rs2734833 has been reported [24,29,43]. 


\section{Clinical characteristics, neuroendocrine factors and gene polymorphisms may predict SSRIs response and remission}

With respect to the HPA and HPT axes, no neuroendocrine indicator independently predicted SSRI response or remission in this study. However, we found the combination of some clinical characteristics, neuroendocrine factors and rs1800544 polymorphisms predicted $74.8 \%$ of the SSRI response and $65.5 \%$ of the SSRI remission. This might provide a valuable way which combined with the genetic pathway, neuroendocrine factors and clinical characteristics may be able to predict SSRI treatment outcome in the future. Our findings were different from previous studies that reported higher TSH independently predicted SSRIs response [44].

Additionally, we found that every one point increase in the HAMD baseline scores was associated with a 0.929 fall in the possibility to research remission ( $\bullet$ Table 5 ) and the remission group had lower baseline HAMD scores relative to the nonremission group. Our finding is consistent with one previous study which stated that higher baseline depressive symptom severity predicted lower probability of remission [45]. In the current study we found that the remission group was younger and had less percent of patients who were married, remarried or divorced relative to the non-remission group. The relationship between age, marital status and antidepressant outcome was also reported by other studies [46].

\section{Limitation}

$\nabla$

There are some limitations of our study. First, we did not measure the plasma levels of the medications. Some studies have shown no relationship between SNPs and plasma drug concentration; however, there may be indirect effects that influence antidepressant efficacy [47]. Second, the relationships between SNPs and side effects were not explored, and neither were relationships with personality and cognitive functions. Further limitations include the relatively short timeframe of the study, the inability to exclude the possibility of a placebo effect because of the lack of a control group and focusing only on a limited number of genetic polymorphisms.

\section{Conclusion}

$\nabla$

In conclusion, despite these limitations, this randomized double-blinded study demonstrates that the responses to SSRIs are significantly associated with catecholamine gene polymorphisms. We concluded that polymorphisms of the DRD4 gene accurately predict SSRI responses in Chinese Han individuals suffering from MDD. Therefore, it will be necessary to replicate and further verify this possibility in other independent studies using larger samples. Independently, neuroendocrine factors were not significant predictors for SSRI outcome, and only their combination with clinical characteristics and gene polymorphisms could predict subject response or remission to SSRIs. Therefore, the influence of gene-environment interactions on SSRI response or remission should be explored in future studies.

\section{Acknowledgements \\ $\nabla$}

We would like to thank our colleagues and volunteers who supported this study. This work was supported by the 973 Program (2010CB529600), the National Key Technology R\&D Program (2004 BA720A21-01, 2012 BAI 01B09, 2009 CB825606), the National Nature Science Foundation of China (81121001, 31171237), the Shanghai Municipal Commission of Science and Technology Program 09DJ1400601, and the Shanghai Leading Academic Discilpine Project (B205), the Sichuan Province Technology R\&D Program (2011FZ0049).

\section{Conflict of Interest}

$\nabla$

The authors declare no conflicts of interest.

\section{Affiliations}

'Department of Psychiatry, West China Hospital of Sichuan University, Chengdu, Sichuan Province, People's Republic of China

${ }^{2}$ Bio-X Institute, Key Laboratory for the Genetics of Developmental and Neuropsychiatric Disorders (Ministry of Education), Shanghai Jiao Tong University, Shanghai, People's Republic of China

${ }^{3}$ Li Yin and Yao Ying Zhang contributed to the work equally

\section{References}

1 Llorca PM, Fernandez JL. Escitalopram in the treatment of major depressive disorder: clinical efficacy, tolerability and cost-effectiveness vs. venlafaxine extendedrelease formulation. Int J Clin Pract 2007; 61: 702-710

2 Machado M, Iskedjian M, Ruiz I et al. Remission, dropouts, and adverse drug reaction rates in major depressive disorder: a meta-analysis of head-to-head trials. Curr Med Res Opin 2006; 22: 1825-1837

3 Trivedi MH, Rush AJ, Wisniewski SR et al. Evaluation of outcomes with citalopram for depression using measurement-based care inSTAR* $D$ : implications for clinical practice. Am J Psychiatry 2006; 163: 28-40

4 Serretti A, Chiesa A, Calati $R$ et al. Common genetic, clinical, demographic and psychosocial predictors of response to pharmacotherapy in mood and anxiety disorders. Int Clin Psychopharmacol 2009; 24: $1-18$

5 Lerer B, Macciardi F. Pharmacogenetics of antidepressant and moodstabilizing drugs: a review of candidategene studies and future research directions. Int J Neuropsychopharmacol 2002; 5: 255-275

6 Jia P, Wang L, Meltzer HY et al. Pathway-based analysis of GWAS datasets: Effective but caution required. Int J Neuropsychopharmacol 2011; 14: 567-572

7 López-León S, Janssens AC, González-Zuloeta Ladd AM et al. Meta-analysis of genetic studies on major depressive disorder. Mol Psychiatry 2008; 13: 772-785

$8 \mathrm{Kim} \mathrm{H,} \mathrm{Lim} \mathrm{SW,} \mathrm{Kim} \mathrm{S} \mathrm{et} \mathrm{al.} \mathrm{Monoamine} \mathrm{transporter} \mathrm{gene} \mathrm{polymor-}$ phisms and antidepressant response in Koreans with late-life depression. JAMA 2006; 296: 1609-1618

9 Yoshida K, Takahashi H, Higuchi $H$ et al. Prediction of antidepressant response to milnacipran by norepinephrine transporter gene polymorphisms. Am J Psychiatry 2004; 161: 1575-1580

10 Bosch OG, Seifritz E, Wetter TC. Stress-related depression: neuroendocrine, genetic, and therapeutical aspects. World J Biol Psychiatry 2012; 13: 556-568

11 aan het Rot M, Mathew SJ, Charney DS. Neurobiological mechanisms in major depressive disorder. CMAJ 2009; 180: 305-313

12 Min W, Liu C, Yang Y et al. Alterations in hypothalamic-pituitary-adrenal/thyroid (HPA/HPT) axes correlated with the clinical manifestations of depression. Prog Neuropsychopharmacol Biol Psychiatry 2012; 39: 206-211

13 Heuser IJ, Schweiger $U$, Gotthardt $U$ et al. Pituitary-adrenal system regulation and psychopathology during amitriptyline treatment in elderly depressed patients and normal comparison subjects. Am J Psychiatry 1996; 15: 93-99

14 Fraser SA, Kroenke K, Callahan CM et al. Low yield of thyroid-stimulating hormone testing in elderly patients with depression. Gen Hosp Psychiatry 2004; 26: 302-309 
15 Brouwer JP, Appelhof BC, Peeters RP et al. Thyrotropin, but not a polymorphism in type II deiodinase, predicts response to paroxetine in major depression. Eur J Endocrinol 2006; 154: 819-825

16 Hamilton $M$. A rating scale for depression. J Neurol Neurosurg Psychiatry 1960; 23 : 56-62

17 Shi YY, He L. SHEsis, a powerful software platform for analyses of linkage disequilibrium, haplotype construction, and genetic association at polymorphism loci. Cell Res 2005; 15: 97-98

18 Barrett JC, Fry B, Maller J et al. Haploview: analysis and visualization of LD and haplotype maps. Bioinformatics 2005; 21: 263-265

19 Li Z, Zhang Z, Tang $W$ et al. A partition-ligation-combination-subdivision EM algorithm for haplotype inference with multiallelic markers: update of the SHEsis (http://analyisis.bio.x.cn). Cell Res 2009; 19: 519-523

20 Willner P. Dopamine and depression: a review of recent evidence. III. The effects of antidepressant treatment. Brain Res 1983; 287: 237-246

21 D'Aquila PS, Collu M, Gessa GL et al. The role of dopamine in the mechanism of action of antidepressant drugs. Eur J Pharmacol 2000; 405: 365-373

22 Willner P, Hale AS, Argyropoulos S. Dopaminergic mechanism of antidepressant action in depressed patients. J Affect Disord 2005; 86: $37-45$

23 Garriock HA, Delgado P, Kling MA et al. Number of risk genotypes is a risk factor for major depressive disorder: a case control study. Behav Brain Funct 2006; 2: 24

24 Serretti A, Zanardi $R$, Cusin $C$ et al. No association between dopamine $\mathrm{D}(2)$ and $\mathrm{D}(4)$ receptor gene variants and antidepressant activity of two selective serotonin reuptake inhibitors. Psychiatry Res 2001; 104: 195-203

25 Dong $C$, Wong $M L$, Licinio J. Sequence variations of ABCB1, SLC6A2, SLC6A3, SLC6A4, CREB1, CRHR1 and NTR K2: association with major depression and antidepressant response in Mexican-Americans. Mol Psychiatry 2009; 14: 1105-1118

26 Min WJ, Ma XH, Li T et al. Association of serotonin and norepinephrine transporter gene polymorphisms with the susceptibility to depression. Zhonghua Yi Xue Yi Chuan Xue Za Zhi 2009; 26: 388-392 [in Chinese]

27 Uher R, Huezo-Diaz P, Perroud N et al. Genetic predictors of response to antidepressants in the GENDEP project. Pharmacogenomics J 2009; 9: $225-233$

28 Baffa A, Hohoff C, Baune BT et al. Norepinephrine and serotonin transporter genes: impact on treatment response in depression. Neuropsychobiology 2010; 62: 121-131

$29 \mathrm{Xu} Z$, Zhang Z, Shi Y et al. Influence and interaction of genetic polymorphisms in catecholamine neurotransmitter systems and early life stress on antidepressant drug response. J Affect Disord 2011; 133: 165-173

30 Fuke S, Suo S, Takahashi N et al. The VNTR polymorphism of the human dopamine transporter (DAT1) gene affects gene expression. Pharmacogenomics J 2001; 1: 152-156

31 Kirchheiner J, Gründemann D, Schömig E. Contribution of allelic variations in transporters to the phenotype of drug response. J Psychopharmacol 2006; 20: 27-32
32 Lavretsky H, Siddarth P, Kumar A et al. The effects of the dopamine and serotonin transporter polymorphisms on clinical features and treatment response in geriatric depression: a pilot study. Int J Geriatr Psychiatry 2008; 23: 55-59

33 Serretti A, Zanardi R, Cusin C et al. Tryptophan hydroxylase gene associated with paroxetine antidepressant activity. Eur Neuropsychopharmacol 2001; 11: 375-380

34 Serretti A, Zanardi R, Rossini $D$ et al. Influence of tryptophan hydroxylase and serotonin transporter genes on fluvoxamine antidepressant activity. Mol Psychiatry 2001; 6: 586-592

35 Arias B, Fabbri C, Gressier $F$ et al. TPH1, MAOA, serotonin receptor 2A and $2 \mathrm{C}$ genes in citalopram response: possible effect in melancholic and psychotic depression. Neuropsychobiology 2013; 67: 41-47

36 Yoshida K, Naito S, Takahashi $H$ et al. Monoamine oxidase: A gene polymorphism, tryptophan hydroxylase gene polymorphism and antidepressant response to fluvoxamine in Japanese patients with major depressive disorder. Prog Neuropsychopharmacol Biol Psychiatry 2002; 26: 1279-1283

37 Peters EJ, Slager SL, McGrath PJ et al. Investigation of serotonin-related genes in antidepressant response. Mol Psychiatry 2004; 9: 879-889

$38 \mathrm{Ham}$ BJ, Lee MS, Lee HJ et al. No association between the tryptophan hydroxylase gene polymorphism and major depressive disorders and antidepressant response in a Korean population. Psychiatr Genet 2005; 15: 229-301

39 Hong CJ, Chen TJ, Yu YW et al. Response to fluoxetine and serotonin $1 \mathrm{~A}$ receptor (C-1019G) polymorphism in Taiwan Chinese major depressive disorder. Pharmacogenomics J 2006; 6: 27-33

40 Kato $M$, Serretti A. Review and meta-analysis of antidepressant pharmacogenetic findings in major depressive disorder. Mol Psychiatry 2010; 15: 473-500

41 Wang HC, Yeh TL, Chang HH et al. TPH1 is associated with major depressive disorder but not with SSRI/SNRI response in Taiwanese patients. Psychopharmacology (Berl) 2011; 213: 773-779

42 Illi A, Setälä-Soikkeli E, Viikki $M$ et al. 5-HTR1A, 5-HTR2A, 5-HTR6, TPH1 and TPH2 polymorphisms and major depression. Neuroreport 2009; 20: 1125-1128

43 Wang $Y$, Liu $X, Y u$ Y et al. The role of single nucleotide polymorphism of D2 dopamine receptor gene on major depressive disorder and response to antidepressant treatment. Psychiatry Res 2012; 200: 1047-1050

44 Brouwer JP, Appelhof BC, Peeters RP et al. Thyrotropin, but not a polymorphism in type II deiodinase, predicts response to paroxetine in major depression. Eur J Endocrinol 2006; 154: 819-825

45 Joel I, Begley AE, Mulsant BH et al. Dynamic prediction of treatment response in late-life depression. Am J Geriatr Psychiatry 2014; 22: 167-176

46 Fournier JC, DeRubeis RJ, Shelton RC et al. Prediction of response to medication and cognitive therapy in the treatment of moderate to severe depression. J Consult Clin Psychol 2009; 77: 775-787

47 Porcelli S, Drago A, Fabbri C et al. Pharmacogenetics of antidepressant response. J Psychiatry Neurosci 2011; 36: 87-113 